\title{
The effect of systemic cocaine on the responses to noxious stimuli and spontaneous activity of medial bulboreticular projection neurons
}

\author{
Carl R. Belczynski Jr. ${ }^{3}$, Antti Pertovaara ${ }^{4, *}$, Thomas J. Morrow ${ }^{2,4}$ and Kenneth L. Casey ${ }^{1,2,4}$ \\ Departments of ${ }^{1}$ Neurology, ${ }^{2}$ Physiology and ${ }^{3}$ Neuroscience Program, University of Michigan, and ${ }^{4}$ Neurology Research Laboratories, \\ VA Medical Center, Ann Arbor, MI 48105 (U.S.A.)
}

(Accepted 13 March 1990)

Key words: Cocaine; Antinociception; Unit analysis; Electrophysiology; Reticular formation; Nociception; Somatosensory

\begin{abstract}
The effect of antinociceptive doses of cocaine $(25 \mathrm{mg} / \mathrm{kg}$, i.p.) on unit responses to noxious somatic stimuli and spontaneous activity of antidromically identified projection neurons in the medial medullary reticular formation (MRF) was studied in the rat. Thirty-three antidromically activated neurons were recorded from the medullary raphe, gigantocellular, or paragigantocellular nuclei in an acute anaesthetized preparation; 25 cells projected to the spinal cord and 8 neurons had rostral projections through the medial forebrain bundle ( $n$ $=4)$ or to the medial thalamus $(n=4)$. After cocaine administration, $24(73 \%)$ of these cells showed immediate $(<5 \mathrm{~min})$ and prolonged (45-70 $\mathrm{min}$ ) increases in their level of spontaneous activity. Associated with this increased interstimulus activity, 21 of 29 (72\%) neurons responsive to noxious somatic stimulation reduced their responsiveness, relative to prestimulus activity, after cocaine administration. In 5 animals tested, the cocaine-induced changes in spontaneous activity and changes in evoked responsiveness were unaffected by naloxone (1 $\mathrm{mg} / \mathrm{kg}$, i.p.) but partially reversed within $5 \mathrm{~min}$ of the administration of chlorpromazine $(3 \mathrm{mg} / \mathrm{kg}$, i.p.). There were no obvious differences in neuronal response characteristics or the effect of cocaine that correlated with anatomical location or direction of axonal projection. Similar results were obtained while recording from 14 somatically responsive units in chronic, unrestrained, lightly anesthetized or awake rats. These findings provide direct evidence that cocaine, in doses that are antinociceptive for the rat, affects both unit responses to noxious stimuli and the spontaneous activity of caudally and rostrally projecting bulboreticular neurons over a time course that parallels the behavioral antinociception. The observation that unit responses to somatic stimuli were reduced while spontaneous activity was unchanged or increased in most cells suggests that cocaine antinociception may be due to the activation of sensory inhibitory mechanisms mediated by the MRF.
\end{abstract}

\section{INTRODUCTION}

Three short reports, published at the turn of the century, described general analgesia produced by the systemic administration of cocaine to $\operatorname{dogg}^{17,29}$ and a human subject ${ }^{15}$. Later studies further suggested that cocaine has antinociceptive ${ }^{33}$ and opiate potentiating effects $^{22}$. Recently, this laboratory demonstrated that systemically administered cocaine is a rapidly acting $(<5$ $\min )$, non-sedative, central analgesic in the $\mathrm{rat}^{20}$ as measured by the hot-plate and the formalin test. As assessed using the formalin test, reduction in pain behavior was shown to be complete and sustained for more than $1 \mathrm{~h}$. The antinociceptive action is not reversible with naloxone, is blocked by dopamine receptor antagonists, and appears to be independent of cardiovascular or local anesthetic effects of the compound ${ }^{20}$.

The CNS mechanisms mediating cocaine-induced antinociception are not known. Recent experiments have shown that antinociceptive doses of cocaine do not suppress nociceptive primary afferent fibers or a spinal nociceptive reflex ${ }^{26}$. Bowsher et $\mathrm{al}^{4}{ }^{4}$ proposed that brainstem reticular neurons coextensive with the nucleus gigantocellularis $(\mathrm{Gi})$ form a relay for nociceptive transmission between the spinal cord and medial thalamic structures. Indeed, many studies have documented evoked activity of bulboreticular neurons to noxious stimuli $^{6,7,8,24,32}$. Thus, cocaine-induced antinociception could be associated with a decrease in the nociceptive responses of rostrally projecting medial bulboreticular neurons or an increase in the activity of bulbospinal neurons that suppress the rostral transmission of nociceptive activity ${ }^{2}$. In the current study, we wished to determine the effect of antinociceptive doses of cocaine on the responses to somatic stimuli of medial medullary reticular formation (MRF) neurons with antidromically identified spinal or diencephalic projections.

\section{MATERIALS AND METHODS}

Acute preparation

Thirty-five adult, male Sprague-Dawley rats (250-375 g) were anesthetized with chloral hydrate $(400 \mathrm{mg} / \mathrm{kg}$, i.p. $)$ and a tracheal

\footnotetext{
*Present address: Department of Physiology, University of Helsinki, Helsinki, Finland.

Correspondence: K.L. Casey, Neurology Service, VA Medical Center, 2215 Fuller Road, Ann Arbor, MI 48105, U.S.A.
} 
cannula was inserted. Supplemental doses of chloral hydrate (100 $\mathrm{mg} / \mathrm{kg}$, i.p.) were given at regular intervals. Animals were paralyzed (Gallamine triethiodide, $55 \mathrm{mg} / \mathrm{kg}$ i.p.) and artificially respirated (T.V. $5 \mathrm{ml}$; R.R. 16-20 bpm). End-tidal $\mathrm{CO}_{2}$ was continuously monitored and maintained between 3.5 and $4.5 \%$. A feedback controlled heating pad kept the rectal temperature between 36.5 and $38.5^{\circ} \mathrm{C}$. Peripheral vascularization was checked by observing the color of the ears, paws and tail. The level of anesthesia was monitored by observing somatic and autonomic (i.e., heart rate, increase in end-tidal $\mathrm{CO}_{2}$ and pupillary dilatation) responses to a noxious pinch sufficient to evoke flexion reflex in lightly anesthetized and awake animals.

Animals were placed in a spinal stereotaxic frame (Kopf) according to the atlas of Paxinos and Watson ${ }^{25}$. In 30 animals, a laminectomy was performed at the level of vertebrae $T_{12}-L_{2}$, the dura removed, and a pool of skin was formed and filled with warm mineral oil. A pair of stainless-steel stimulating electrodes was placed bilaterally in the dorsal lateral funiculi of the spinal cord. In these and 5 additional animals, the skull was exposed and 4 holes were drilled for placement of a recording electrode, a reference electrode, and two Teflon-coated, twisted bipolar stimulating electrodes. The latter were aimed bilaterally at the medial forebrain bundle (MFB coordinates: A $6.44 \mathrm{~mm}, \mathrm{~L} \pm 2.0 \mathrm{~mm}, \mathrm{D} 1.6 \mathrm{~mm} ; 22$ animals) or at the medial thalamic nucleus parafascicularis (PF coordinates: A $4.8 \mathrm{~mm}, \mathrm{~L} \pm 1.2 \mathrm{~mm}, \mathrm{D} 4.0 \mathrm{~mm} ; 13$ animals).

Unit activity was recorded extracellularly with Epoxylite-coated stainless-steel microelectrodes (tip impedance $8-20 \mathrm{M} \Omega$ at $100 \mathrm{~Hz}$; coordinates: P $2.6 \mathrm{~mm}, \mathrm{~L} 0.0 \mathrm{~mm}, \mathrm{D} \pm 0.5 \mathrm{~mm}$ ) then amplified and filtered using standard techniques. Action potentials from single neurons were isolated using a spike window discriminator; the triggered pulse output, along with digitized outputs from all stimulating devices were fed to the inputs of a minicomputer system. Stimuli included transcutaneous electrical (5-10 mA, 5 ms duration, $0.2-0.4 \mathrm{~Hz}$ ), peripheral mechanical (toothed forceps, Von Frey hairs, and airpuff) and orthodromic or antidromic CNS electrical stimuli (spinal cord, diencephalon and MFB; 0.1-4.0 mA, $0.1 \mathrm{ms,}$ $0.2-0.4 \mathrm{~Hz}$ ).

During the search for neurons, the recording electrode was advanced with a microdrive while the lumbar cord was stimulated (0.1 ms square pulse, $0.4-4 \mathrm{~mA}, 0.2-0.4 \mathrm{~Hz}$ ). The rostral pair of stimulating electrodes ipsilateral to the recording electrode was also activated $(0.1 \mathrm{~ms}, 0.1-1.5 \mathrm{~mA}, 0.25 \mathrm{~Hz})$ and peripheral stimuli were delivered to the body with each $100 \mu \mathrm{m}$ movement of the recording electrode. We were thus able to locate and test those units that were not spontaneously active or were lacking an evoked response. Neurons were tested for antidromic activation by the collision method $^{11}$ as shown in Fig. 1 and fully characterized with respect to adequate stimuli, stimulus response and receptive field. The effect of cocaine was studied only when neurons had an antidromically identified projection.

Computer software developed in this laboratory was used for the acquisition, storage and analysis of all unit and stimulation data. In addition to compiling single trial or normalized, multiple-trial, peri-stimulus time histograms (PSTHs), the program computes statistical parameters that characterize each PSTH. Relative stimulus evoked responses were computed as the difference between the area of the histogram during the response period and the area of the histogram during an equivalent time segment of the prestimulus period.

Once a neuron was selected, normalized PSTHs were generated from multiple trials of constant intensity somatic stimuli. Histograms of spontaneous unit activity were also generated. Cocaine- $\mathrm{HCl}$ was then administered at a dose of $25 \mathrm{mg} / \mathrm{kg}$ i.p. in sterile saline. This dose produces antinociception in behavioral studies ${ }^{20}$. Multiple-trial PSTHs and histograms of spontaneous activity were again generated, at regular intervals, for up to $90 \mathrm{~min}$ after cocaine administration for comparison with pre-cocaine controls. In some experiments, naloxone ( $1 \mathrm{mg} / \mathrm{kg}$, i.p.) and chlorpromazine ( $3 \mathrm{mg} / \mathrm{kg}$, i.p.) were given, in saline vehicle, 15 and $40 \mathrm{~min}$ after cocaine, respectively. In one experiment, neuronal responsiveness to noxious electrical forepaw shock was tested at various time intervals after an antidromic conditioning train stimulus ( 6 pulses, 20 ms duration, 250 $\mathrm{Hz}$ ) was delivered to the spinal cord. After testing was completed (approx. 1.3 hours) electrolytic lesions (35-50 $\mu \mathrm{A} \mathrm{DC}$ current for 20 s) were made at recording and stimulation sites for histological verification. The animals were given an anesthetic overdose and perfused with $10 \%$ formalin; $60 \mu \mathrm{m}$ sections were stained with Cresyl violet and all structures were identified as labeled in the atlas of Paxinos and Watson ${ }^{25}$.

\section{Chronic preparation}

Four chronic recording animals were prepared as described previously ${ }^{23}$. During surgery, a chronic recording mount was positioned stereotaxically above the MRF (P $2.6 \mathrm{~mm}, \mathrm{ML} 0.0 \mathrm{~mm}$ ) and cemented to the skull. Stimulating electrodes were placed bilaterally in the MFB (A $6.44 \mathrm{~mm}, \mathrm{ML} 2.0 \mathrm{~mm}$ ) or the medial thalamic CM-PF complex (A $4.84 \mathrm{~mm}$, ML $1.4 \mathrm{~mm}$ ) for antidromic stimulation. Animals were allowed 2 weeks to recover before recording was initiated. Before a recording session, chloral hydrate $(320 \mathrm{mg} / \mathrm{kg}$, i.p.) was administered and an Epoxylite-coated stainless-steel electrode was inserted to a depth 1-2 mm above the dorsal MRF. Recording was differential relative to a reference electrode implanted in frontal cortex at the time of surgery. The electrode was connected to the amplifier via a mercury commutator (Dragonfly Industries) mounted above the recording chamber to minimize cable and movement artifacts. At the time of neuronal testing, all animals exhibited a robust flexion reflex to hindpaw pinch. Unit recording, stimulation, and data analysis were conducted as described above.
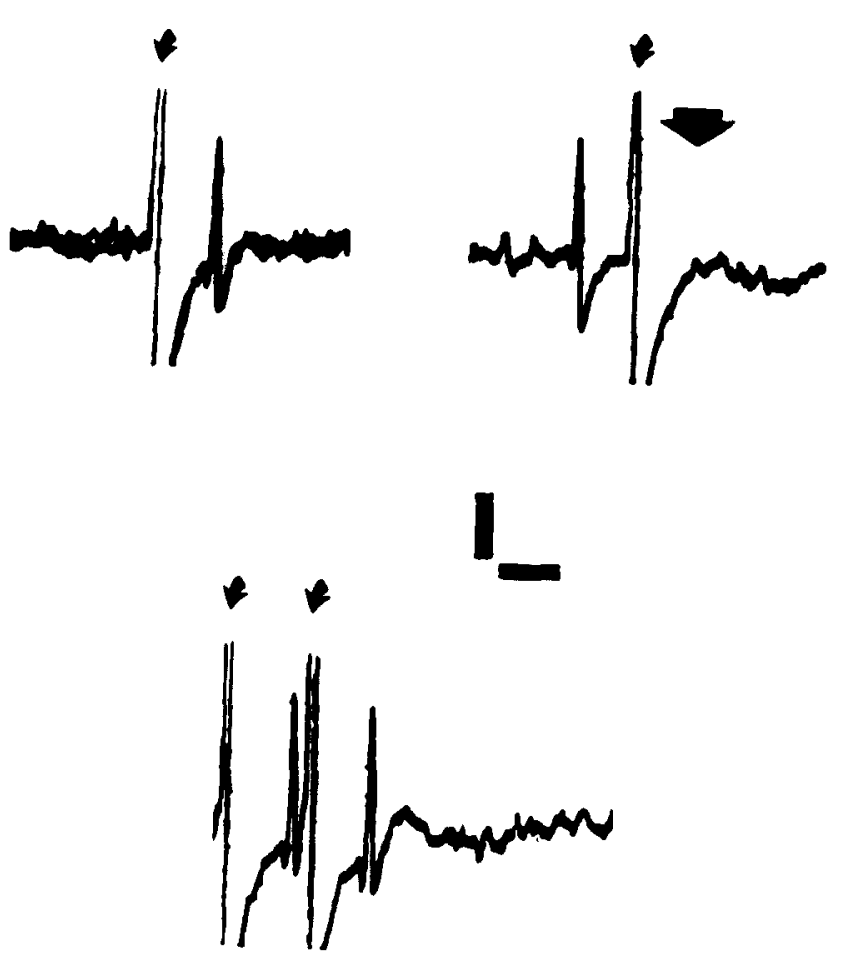

Fig. 1. Medial bulboreticular neuron antidromically activated from the lumbar spinal cord. Upper left: 3 traces superimposed showing latency variation of $<0.2 \mathrm{~ms}$ (small arrow = stimulus artifact). Upper right: single trace showing disappearance of antidromic spike (large arrow) after collision with spontaneous orthodromic spike. Bottom: twin pulse stimulation (isi $=2.2 \mathrm{~ms}$ ) demonstrating high frequency following. Vertical bar $=100 \mu \mathrm{V}$, horizontal bar $=1 \mathrm{~ms}$. 
RESULTS

\section{Acute preparation}

Two-hundred and seventy-four MRF neurons were characterized with respect to adequate stimulus and receptive field and were tested for antidromic activation. Units responded primarily to noxious stimuli and demonstrated large, often bilateral, receptive fields that typically covered one or more limbs. Thirty-three of these neurons could be antidromically activated and were tested with cocaine. Only a single neuron per animal was completely studied because of the uncertainty about the duration of the effect of the intraperitoneally administered drugs. Twenty-five of these neurons were antidromically activated from the lumbar cord, 4 were driven from the MFB and 4 from the medial thalamus. Lesions at recording sites were histologically verified in 30 animals and were confined to the medial aspect of the reticular formation near the pontomedullary junction (Fig. 2). Rostral stimulating electrode placements were verified in all cases; medial thalamic structures stimulated were nucleus parafascicularis ( 2 animals) and centralis lateralis ( 2 animals). No obvious anatomical segregation of MRF neurons correlated with the direction of axonal projection.

Within $5 \mathrm{~min}$ of cocaine administration, 73\% (24/33) of the neurons showed a marked increase and $12 \%$ (4/33) showed no change in the level of spontaneous activity (Fig. 3). Seventy-two percent (18/25) of the spinally projecting neurons showed increases in spontaneous activity after cocaine, whereas $75 \%(6 / 8)$ of the spontaneously active rostral projection neurons increased their level of activity. Before cocaine administration, caudally projecting neurons demonstrated an average spontaneous firing rate of $5.5 \mathrm{~Hz}$ (range: $0.25-14 \mathrm{~Hz}$ ). Increases in spontaneous activity for caudally projecting neurons averaged $21 \mathrm{~Hz}(1600 \%)$, with post-cocaine rates ranging from 0.35 to $104 \mathrm{~Hz}$. Four caudally projecting neurons decreased their spontaneous firing rate after cocaine from an average of $12 \mathrm{~Hz}$ (range; 3-26 Hz) to $5 \mathrm{~Hz}$ (range; 0.4-14 Hz). Rostrally projecting neurons demonstrated an average spontaneous firing rate of $0.55 \mathrm{~Hz}$ (range; 0.1-1 Hz) before cocaine administration. The 4 cells antidromically activated from the MFB showed an average increase of $14 \mathrm{~Hz}$ (range; $2-30 \mathrm{~Hz}$ ) after cocaine, whereas only two cells projecting to the medial thalamus showed increases in spontaneous activity of 8 and $2.3 \mathrm{~Hz}$. Of the remaining two neurons projecting to the medial thalamus, one showed no spontaneous firing before or after cocaine whereas the other demonstrated a decrease of $0.05 \mathrm{~Hz}(11 \%)$ in spontaneous activity after cocaine administration. All values were computed from the maximal changes in activity. The changes in spontaneous activity were of durations ranging from 45 to $70 \mathrm{~min}$. Fig. 4 shows an example of the magnitude and timing of observed increases in spontaneous firing rate for both rostrally and caudally projecting units. The neuron in Fig. 4A was antidromically activated from the ipsilateral MFB; the neuron in Fig. 4B from the lumbar spinal cord. The neuron in Fig. 4A also shows a strong bursting pattern that emerges after cocaine administration; this was observed during the study of one other rostrally projecting neuron.

Twenty-nine neurons demonstrated responses to somatic stimuli before cocaine administration. Nearly all (28/29) of the neurons responded exclusively or differentially to pinching of the skin with toothed forceps; many (23/29) also responded to high intensity transcutaneous electrical stimulation. A small proportion (5/29) of the neurons discharged to innocuous as well as noxious stimuli. Only one neuron responded exclusively to innocuous stimulation (hair movement).
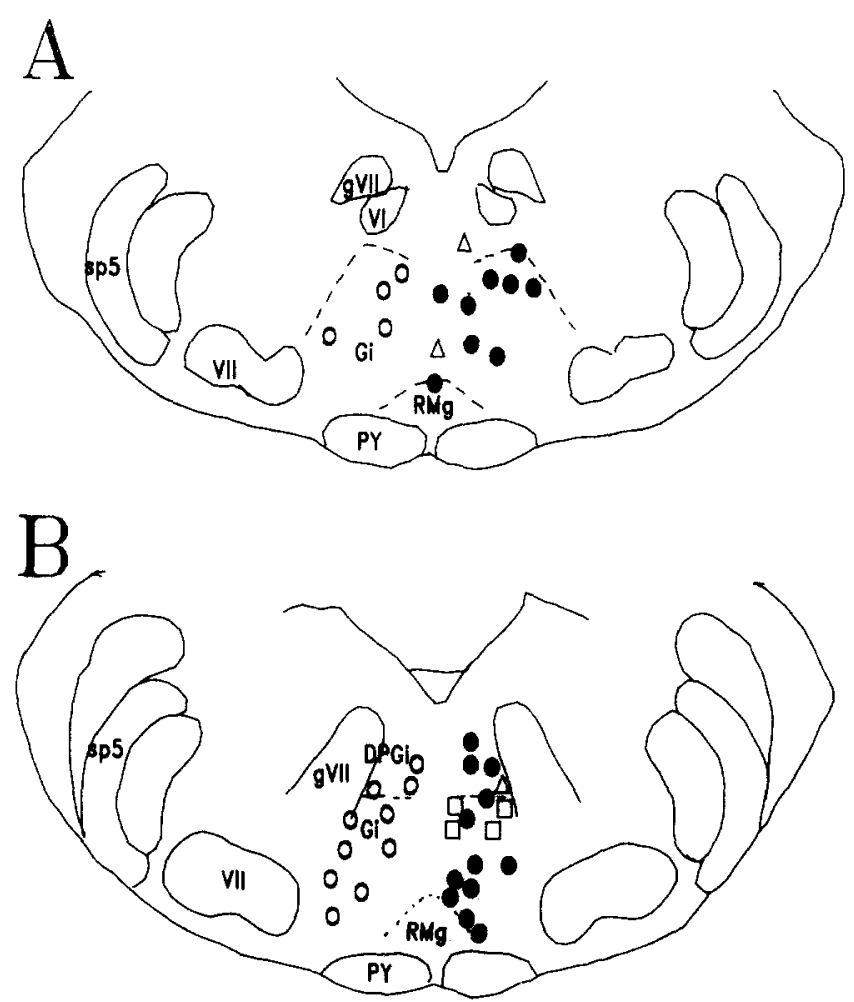

Fig. 2. Anatomical distribution of recorded sites. A and B represent composite coronal sections through the medullary brainstem (modified from Paxinos and Watson ${ }^{26}$ ). $\mathrm{A}$ is $0.8 \mathrm{~mm}$ caudal to $\mathrm{B}$. MRF projection neurons from acute animals are plotted on the right; those recorded in the chronic preparation are on the left. Spinally projecting cells are indicated with filled circles, while neurons with ascending projections are subdivided into those projecting to medial thalamus (open rectangles) or through the medial forebrain bundle (open triangle). Open circles represent location of cells recorded in chronic animals. PY, pyramidal tract; sp5, spinal trigeminal nucleus; VI, abducens nucleus; VII, facial nucleus; gVII, genu of facial nerve; $\mathrm{Gi}$, nucleus gigantocellularis; DPGi, dorsal nucleus paragigantocellularis; $\mathrm{RMg}$, nucleus raphe magnus. 
Excitatory responses to somatic stimuli were elicited from 24 neurons. Neurons with sufficient background firing rates could show additional inhibitory responses to stimulation (4 neurons) or inhibitory components of a biphasic (excitatory and inhibitory) response (6 neurons). Only one of the neurons with a biphasic response projected rostrally to the thalamus. In 3 cases, identical stimuli delivered to different body regions elicited opposite responses. Only a single neuron was found with a purely inhibitory response to nociceptive stimulation.

The most frequently observed effect of cocaine on neural discharge was to reduce unit excitatory responses to somatic stimulation as compared with the prestimulus (background) period. Relative responses were computed as the difference in neuronal discharge between the area of the histogram during the poststimulation period and the area of the histogram during an equivalent time segment of the prestimulus period. Relative responses to stimuli after cocaine administration were reduced compared to the responses before cocaine was given. This relative response suppression was observed during the study of $16 / 22(73 \%)$ of the neurons that projected caudally and 5/7 (71\%) of neurons projecting rostrally (Fig. 3). The average decrease in relative response was 65\% (range: $17-100 \%$ ) of pre-cocaine control levels for caudally projecting cells and $81 \%$ (range: $33-100 \%$ ) for rostrally projecting neurons. Sixteen caudally projecting

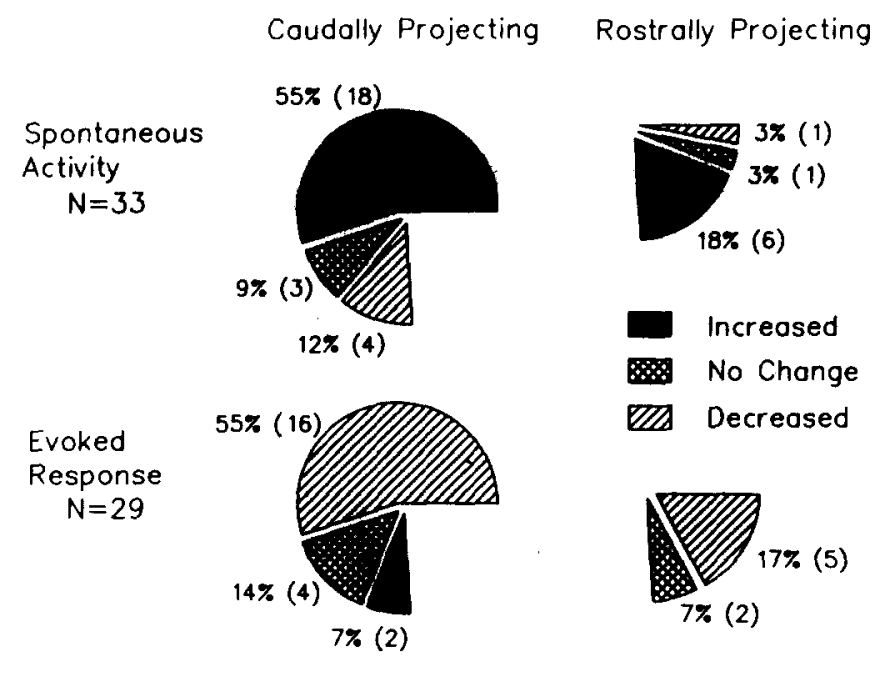

Fig. 3. Schematic summary of cocaine-dependent changes in spontaneous activity (33 neurons) and unit responsiveness to somatic stimuli (29 neurons) of MRF neurons projecting either to the spinal cord or rostrally to the MFB or medial thalamus. The majority of neurons (numbers shown in parentheses) showed increased levels of spontaneous activity and a decrease in the relative excitatory response to noxious stimuli after cocaine. Percentages are computed with respect to cell totals in each row. Number of neurons is indicated in parentheses. Solid rectangle = increased activity or response; diagonal hatched rectangle = decreased activity or response; cross-hatched rectangle $=$ no change in activity or response. cells showed an average decrease of 55\% (range: $22-$ $100 \%$ ) in response levels, and 5 of the rostrally projecting cells showed an average decrease of $45 \%$ (range: 4$100 \%$ ) in their absolute evoked response. Two caudally projecting cells showed increased excitatory responses to stimuli (75\% and $34 \%$ ); one of these increased spontaneous firing whereas the other showed no change in background activity after cocaine. Fig. 5 shows examples of relative response suppression for two rostrally projecting neurons that responded to noxious mechanical stimulation and one caudally projecting neuron with a response to innocuous tactile stimulation. In each case, multiple trial histograms were generated to identical stimuli $5 \mathrm{~min}$ before (control) and $5 \mathrm{~min}$ after administration of cocaine. In 5 animals, the effect of cocaine was not antagonized by naloxone, but could be partially reversed within $5 \mathrm{~min}$ of administration of the nonselective dopamine receptor antagonist, chlorpromazine (Fig. 6).

The finding that an increased spontaneous activity of
A
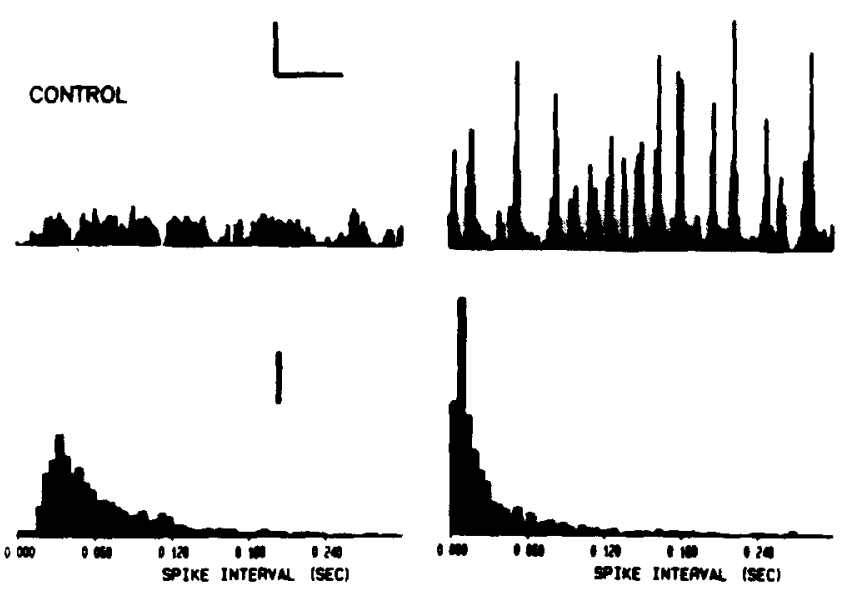

8

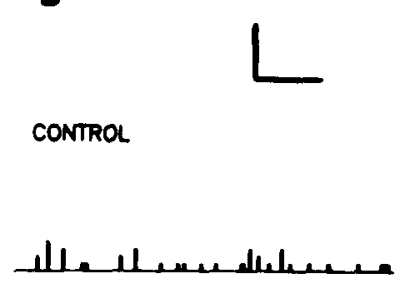

COCNNE

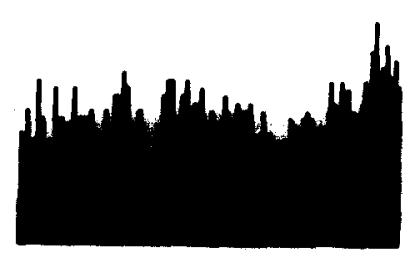

Fig. 4. A: single-trial spontaneous activity histograms (top) of a rostrally (MFB) projecting MRF neuron before and $5 \mathrm{~min}$ after cocaine administration. Corresponding interspike interval histograms are shown immediately below. Mean spike frequency increased from 13.2 to $22.2 \mathrm{~Hz}$ after cocaine administration. Scale bars: upper - vertical $=20$ spikes, horizontal $=20 \mathrm{~s}$, binsize $=500$ $\mathrm{ms}$; lower - vertical $=60$ spikes/interval, intervals $=1500$. B: single-trial histograms showing spontaneous activity of a caudally projecting MRF neuron before and $5 \mathrm{~min}$ after cocaine. Mean spike frequency significantly increased from 0.4 to $29.6 \mathrm{~Hz}$. Vertical bar $=5$ spikes, horizontal bar $=15 \mathrm{~s}$, binsize $=500 \mathrm{~ms}$. 
MRF neurons produced decreased somatosensory responses relative to background activity suggested an MRF-induced suppression of excitatory somatic inputs. This possibility was tested in one experiment in which the tetanic antidromic activation of an MRF cell was associated with a suppression of responsiveness of that neuron to a somatic stimulus. Fig. 7 demonstrates the effect of a conditioning, antidromic, electrical train stimulus of the spinal cord on an MRF neuronal response to cutaneous stimulation. Shortening of the conditioningtesting (C-T) interval to $10 \mathrm{~ms}$ results in complete abolition of the unit response to the cutaneous stimulus (Fig. 7C). The neuron became less responsive to the
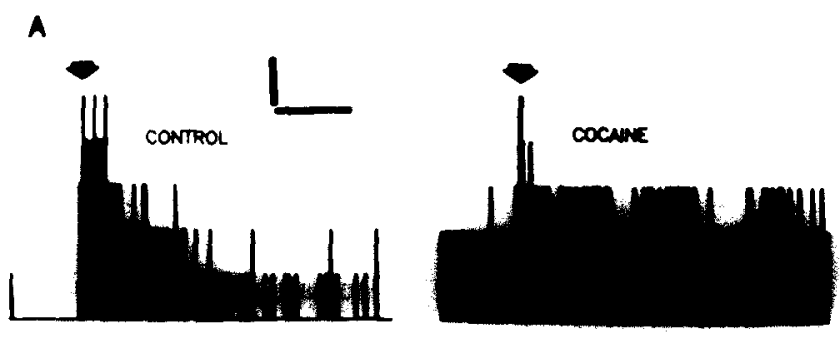

$\mathbf{B}$
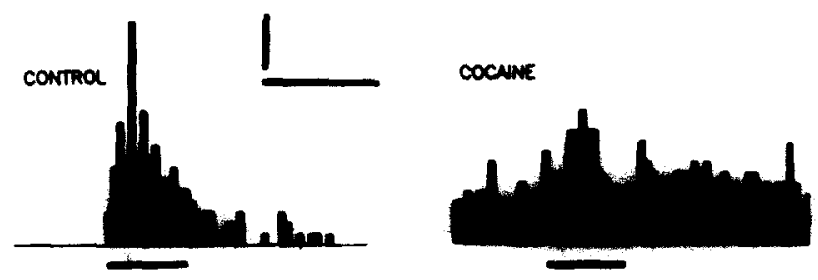

c

control

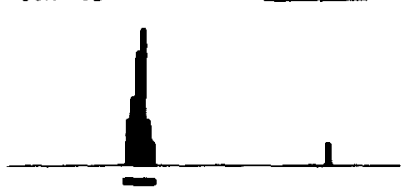

COCANE

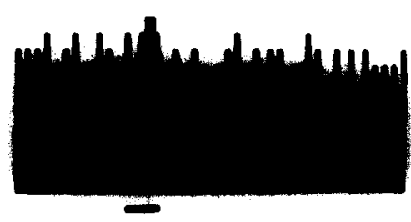

Fig. 5. Cocaine-induced increases in prestimulus activity $(P<0.01)$ with simultaneous reduction of evoked excitatory responses relative to the prestimulus period. A: multiple-trial peri-stimulus time histograms (PSTHs) for an MRF neuron responding to a single ipsilateral earpinch (large arrow) and projecting through the MFB. Vertical bar $=1$ spike/stimulus, horizontal bar $=5 \mathrm{~s}$, binsize $=100$ ms. B: multi-trial PSTHs for an MRF neuron activated by noxious pinch of the face (stimulus bar, 10 trials) and projecting to the medial thalamus on the ipsilateral side. Vertical bar $=0.5$ spikes/stimulus, horizontal bar $=1 \mathrm{~s}$, binsize $=50 \mathrm{~ms}$. C: response of a spinally projecting MRF neuron to innocuous airpuff stimulation (stimulus bar, 10 trials) before and $10 \mathrm{~min}$ after cocaine. Prestimulus activity increased significantly from 0 to $15.6 \mathrm{~Hz}(\mathrm{p}<$ 0.01 ) while the relative excitatory response was suppressed. Histograms are normalized with respect to number of stimulus trials. Vertical bar $=0.4$ spikes/stimulus, horizontal bar $=1 \mathrm{~s}$, binsize $=$ $50 \mathrm{~ms}$.
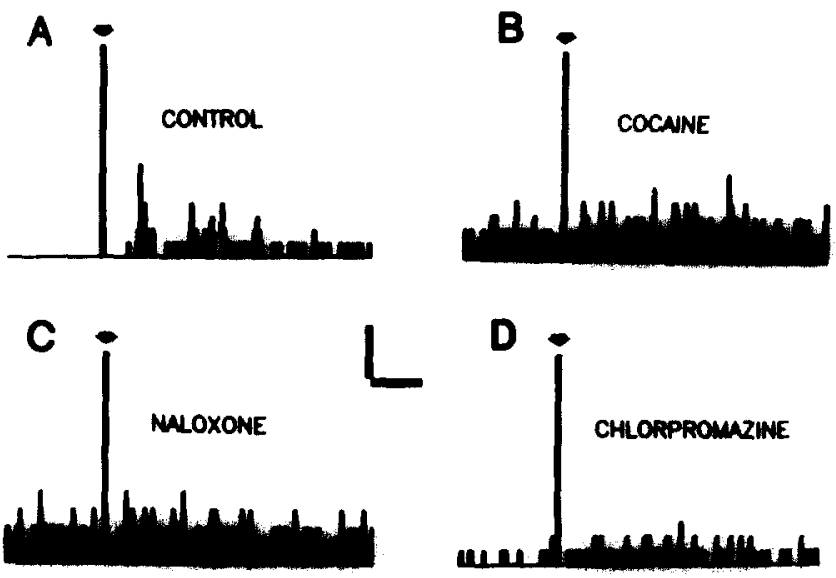

Fig. 6. Response of a caudally projecting neuron to transcutaneous electrical stimulation of the ipsilateral forepaw $(10 \mathrm{~mA}, 5 \mathrm{~ms}, 0.20$ $\mathrm{Hz}, 15-18$ trials). Arrow designates stimulus artifact. A,B: cocaine induced a significant increase $(5 \mathrm{~min})$ in prestimulus (background) activity $(t=17.4, P<0.01)$ with a concomitant reduction in the excitatory response relative to prestimulus activity. The increase in prestimulus activity and reduction in evoked response were partially reversed by chlorpromazine (D, $3 \mathrm{mg} / \mathrm{kg}$, i.p.) but not by naloxone (C, $1 \mathrm{mg} / \mathrm{kg}$, i.p., these histograms were taken 15 (C) and 25 (D) min after cocaine administration and 5 min after drug administration). All histograms are normalized with respect to the number of stimulus trials. Vertical bar $=0.2$ spikes/stimulus, horizontal bar $=$ $100 \mathrm{~ms}$.

cutaneous stimulus after cocaine injection (Fig. 7D).

Cocaine also unmasked (enhanced) inhibitory unit responses relative to prestimulus activity. Neurons that
A

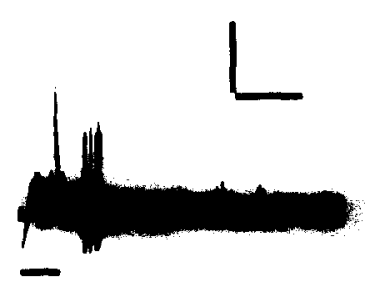

C

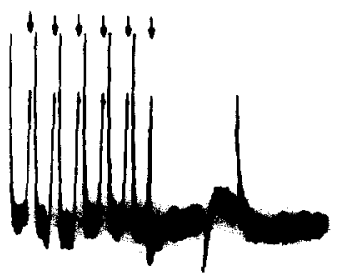

B

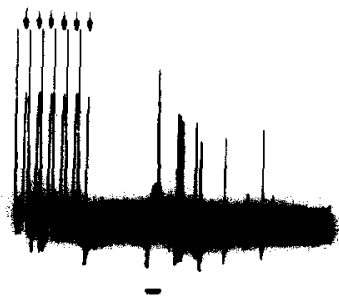

D

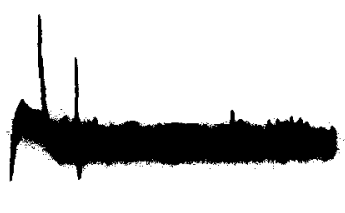

Fig. 7. Antidromic conditioning train $(1.2 \mathrm{~mA}, 250 \mathrm{~Hz}, 6$ stimuli) produces post-tetanic inhibition of excitatory cutaneous inputs to an MRF neuron. Arrows denote antidromic spikes elicited during conditioning train; bars indicate artifact from electrical forepaw test stimulus $(15 \mathrm{~V}, 5 \mathrm{~ms}$ duration, $0.25 \mathrm{~Hz}$ ). A: in the absence of a conditioning antidromic train there is a robust response to electrical cutaneous forepaw stimulus. Scale bars $=10 \mathrm{~ms}, 0.4 \mathrm{mV}$. B: $20 \mathrm{~ms}$ after conditioning train of 6 antidromic spikes, forepaw test stimulus continues to produce neuronal response. Scale bars $=20 \mathrm{~ms}, 0.4$ $\mathrm{mV}$. C: $10 \mathrm{~ms}$ after conditioning train, response to forepaw test stimulus is abolished. Scale bars $=10 \mathrm{~ms}, 0.4 \mathrm{mV}$. D: decreased response to forepaw test stimulus after cocaine. Scale bars $=10 \mathrm{~ms}$, $0.4 \mathrm{mV}$. 


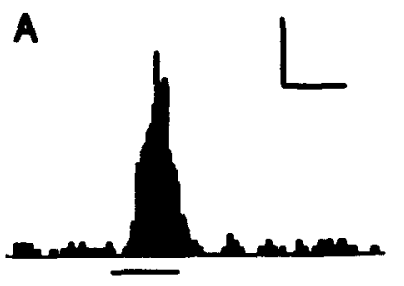

B

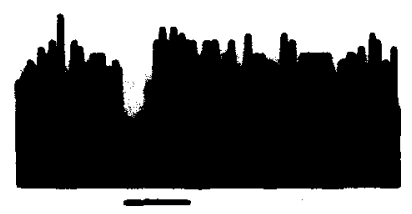

C

D
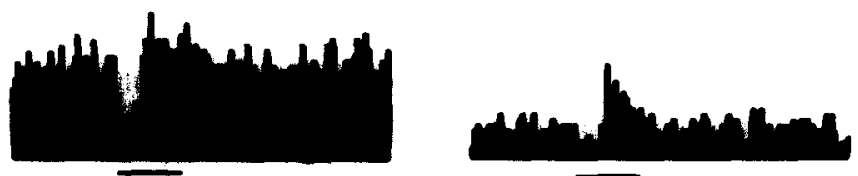

Fig. 8. PSTHs of a caudally projecting MRF neuron showing either an excitatory $(A)$ or inhibitory response $(B, C)$ to noxious earpinch (stimulus bar, 7-10 trials). Numbers indicate the time after cocaine injection in minutes. Cocaine significantly increased prestimulus activity at $5(\mathrm{~B})$ and $30(\mathrm{C}) \mathrm{min}(P<0.01)$ with a corresponding relative reduction in the excitatory phase of the response. Concurrent with the relative suppression of excitatory activity, an inhibitory phase of the unit response is unmasked. The excitatory response and level of prestimulus activity began to recover 75 (D) min after cocaine injection. All histograms are normalized for the number of stimulus trials. Vertical bar $=2$ spikes/stimulus, horizontal bar $=1$ $\mathrm{s}$, binsize $=50 \mathrm{~ms}$.

demonstrated both excitatory and inhibitory responses to noxious stimuli showed both effects of cocaine simultaneously; the inhibitory response became more apparent while the relative excitatory response was reduced. Fig. 8 shows an example of a neuron that responds to noxious pinch delivered anywhere on the body surface. Before cocaine administration, an inhibitory response cannot be detected because of the low level of prestimulus activity. After cocaine administration, inhibition became apparent as prestimulus activity increased and the excitatory response disappeared. Similar effects were seen in 3 neurons with complex heterotopic responses. Fig. 9, for example, shows a neuron that exhibited an excitatory response to forepaw pinch and an inhibitory response to pinching the face. Cocaine increased the level of spontaneous activity and decreased the relative excitatory response while the inhibitory response became more obvious. Naloxone did not reverse the effect of cocaine on either response, but chlorpromazine reduced prestimulus activity and partially restored the excitatory response 5 min after administration.

\section{Chronic preparation}

We considered that the increased spontaneous activity we observed might be due to a cocaine-induced arousal effect appearing against a background of deep chloral hydrate anesthesia. In addition, we wished to examine the possibility that nociceptive inputs from the stereotaxic frame and surgical wound might, during a relatively aroused state, attenuate nociceptive responses by activating diffuse noxious inhibitory controls (DNICs) ${ }^{19}$. Accordingly, 14 of 104 characterized cells were stimulusactivated and tested with cocaine in 4 chronically prepared animals. A minimum of one week was allowed between cocaine administrations in multiply injected animals. Nine units were recorded from rats lightly anesthetized with chloral hydrate and allowed to recover to a level at which flexion reflexes were easily elicited and spontaneous movements appeared. The locomotor effects of cocaine made the routine use of unanesthetized subjects impractical. Ninety-two percent (13/14) of the neurons demonstrated an increase in spontaneous firing rate within 5 min of cocaine administration. The average

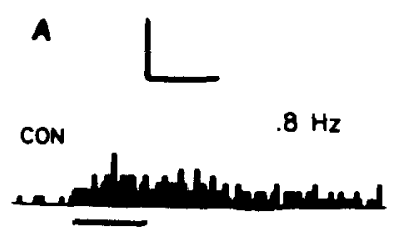

$\operatorname{coc}$

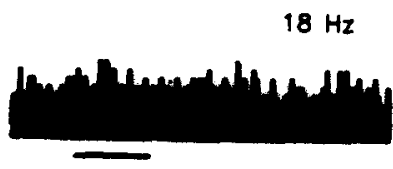

ML

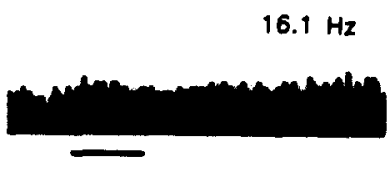

CPZ

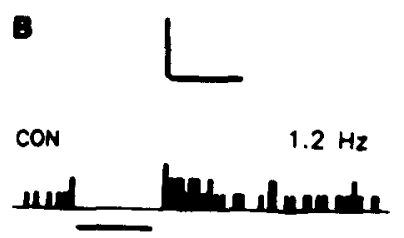

$\operatorname{coc}$
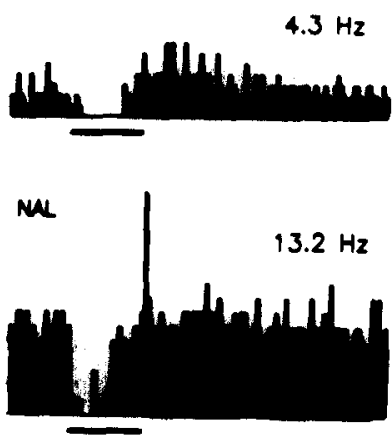

CPZ
$.4 \mathrm{~Hz}$

$\mathrm{O} \mathrm{Hz}$

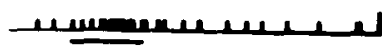

Fig. 9. PSTHs of a caudally projecting MRF neuron showing an excitatory response to noxious pinch of the forepaws (A) and an inhibitory response to pinch of the face (B). Each vertical series of histograms shows evoked responsiveness before cocaine (CON), 5 min after cocaine (COC), 5 min after naloxone (NAL) and 5 min after chlorpromazine (CPZ). Numbers refer to trials given and mean prestimulus firing rate in $\mathrm{Hz}$. Prestimulus activity is significantly increased $(P<0.01)$ after cocaine; this effect is not reversed by naloxone but the activity is reduced to control levels or below after chlorpromazine. The relative excitatory response in column $A$ is suppressed after cocaine and naloxone but partially recovers after chlorpromazine while the relative inhibitory response in $B$ is enhanced after cocaine and naloxone but not measurable after chlorpromazine. The apparent difference between NAL (A) and NAL (B) is that the vertical scale in column B is 0.5 spikes/stimulus, half that of $A$. Horizontal scale in both $A$ and $B=1 \mathrm{~s}$. All histograms are normalized for the number of trials. Binsize $=50 \mathrm{~ms}$. 
level of spontaneous activity before cocaine administration was $29 \mathrm{~Hz}$ (range: 1-150 Hz). The average increase after cocaine was $25 \mathrm{~Hz}(178 \%)$, ranging from 1.4 to 58 $\mathrm{Hz}$. All of the cells recorded responded to stimulation of the body surface, most often to tap stimuli in the hindquarter area, but also to brush, noxious pinch, and electrical tail stimulation $(10 \mathrm{~mA}, 5 \mathrm{~ms}, 0.25 \mathrm{~Hz})$. Receptive fields were large and bilateral. Seventy-one percent (10/14) of the cells showed an average relative decrease of $69 \%$ (range: $33-100 \%$ ) in stimulus related activity compared to the prestimulus period. Two cells exhibited inhibitory responses to somatic stimuli; one had the response obliterated after cocaine, and one other showed an enhanced response. None of the cells showed biphasic responses as was observed in the acute, deeply anesthetized preparation. All 5 of the cells recorded from completely awake animals increased their spontaneous firing rate after cocaine. Three of these 5 cells showed reduced responses to somatic stimuli relative to background. Fig. 2 shows that 9 cells could be localized to the $\mathrm{Gi}$ and 3 to the dorsal nucleus paragigantocellularis (DPGi).

\section{DISCUSSION}

This study shows that antinociceptive doses of cocaine reduce nociceptively-evoked responses of projection neurons in the medial medullary reticular formation for a duration that parallels the antinociception seen in awake rats $^{20}$. The effect was observed in neurons located in several midline reticular nuclei: nucleus reticularis gigantocellularis (Gi), nucleus raphe magnus ( $\mathrm{RMg}$ ) and dorsal nucleus paragigantocellularis (DPGi). There was no obvious difference in the neuronal response characteristics or the effect of cocaine that correlated with anatomical location or with the direction of axonal projection, although all neurons projecting to the medial thalamus were localized in DPGi. The complexity of receptive fields and neuronal responses we observed are consistent with previous reports ${ }^{6,7,8,24,32}$. Our percentage of rostrally projecting neurons $(24 \%)$ is biased by design; others have reported unbiased estimates in the range of $9-13 \%^{8,16,21}$.

The results of the chronic preparations are in general agreement with those from the acute experiments. Neurons within the brainstem responding to somatic stimuli demonstrated increases in spontaneous firing frequency with concomitant decreases in their stimulus evoked responses relative to prestimulus periods. This was the case whether animals were completely awake or lightly anesthetized. Although we could not determine whether the neurons we recorded from these chronically prepared rats had rostral or caudal projections, the neurons exhibited response and receptive field properties that were similar to those recorded from the anesthetized preparations. In any case, anesthesia cannot account for the results observed in the anesthetized rats, nor does it appear that there is an interaction between the anesthetic and cocaine that is responsible for the observed effects of cocaine.

Cocaine appears to be acting within the CNS via a dopaminergic, non-opiate mechanism. In both the behavioral study of Lin et al. ${ }^{20}$ and the present study, the non-selective dopamine receptor antagonist chlorpromazine reversed the effects of cocaine. The opiate receptor antagonist, naloxone, was ineffective in both studies ${ }^{20}$. However, we cannot rule out the possibility that other monoaminergic systems (i.e. norepinephrine, serotonin) are involved in eliciting cocaine's effects on MRF neurons. Cocaine is known to inhibit central monoamine reuptake of noradrenergic ${ }^{1,13}$, serotonergic ${ }^{18,30}$ and dopaminergic ${ }^{12,14}$ systems. Pitts and Marwah $^{28}$ have shown that, in addition to suppressing spontaneous firing of dopaminergic neurons within the substantia nigra (SN) and ventral tegmental area (VTA), systemic cocaine profoundly suppresses the spontaneous firing of noradrenergic neurons of the locus coeruleous (LC) and, to a lesser extent, serotonergic neurons of the dorsal raphe nucleus (DRN). These effects were demonstrated with intravenous doses as low as $0.25 \mathrm{mg} / \mathrm{kg}$. Although we have not measured the blood levels of cocaine achieved in the current study, it is likely that the dose of cocaine we used influenced other monoaminergic neurons. Given the widespread distribution of terminal axons of the noradrenergic neurons of the $\mathrm{LC}^{31}$, it is possible that the changes in neuronal discharge we observed were due in part to the effect of cocaine on these or other catecholaminergic neurons.

It is unlikely that the changes in neuronal discharge observed in our experiments were due to local anesthetic or cardiovascular effects of cocaine. Pertovaara et al. ${ }^{26}$ have shown that systemic doses of cocaine do not block impulse conduction in small diameter peripheral nerve fibers or suppress a nociceptive spinal reflex discharge; and cocaine at doses up to $4 \mathrm{mg} / \mathrm{kg}$ i.v. did not decrease the amplitude or slope of neuronal action potentials ${ }^{28}$. The effects of cocaine on arterial pressure are of short duration ( $<1 \mathrm{~min}$ ) and are not sufficient to induce the analgesia associated with reflex bradycardia ${ }^{20,27}$. Furthermore, procaine, a structurally related local anesthetic, induces a similar cardiovascular response without a similar neurophysiological effect ${ }^{27}$.

We can only speculate as to the possible neurophysiological mechanisms underlying the effect of cocaine on MRF neuronal responses. The neurophysiological action of cocaine on MRF neurons cannot be attributed to a 
generalized depression of neuronal excitability because these neurons demonstrated an increase in spontaneous firing rate. Likewise, a generalized suppression of synaptic inputs to MRF neurons is not consistent with the persistence of noxious-evoked inhibitory responses to noxious stimuli after cocaine administration. The increases in spontaneous activity (up to $100 \mathrm{~Hz}$ ) were of insufficient magnitude to exceed biophysical limitations on neuronal firing frequencies (see Fig. 7). It is possible that collaterals of MRF neurons could inhibit selective excitatory inputs during periods of high activity. This mechanism would not reduce the efficacy of strong inhibitory inputs, which would become more apparent while the cell's activity is high. We have demonstrated that antidromically induced repetitive neuronal activity can produce a suppression of excitatory cutaneous input and Brodal $^{5}$ has presented anatomical evidence for the existence of recurrent axon collaterals in MRF projection neurons. It is also possible that the mechanism of response suppression may involve post-synaptic inhibition of excitatory sensory inputs to distal dendrites of MRF cells. Further experiments will be necessary to elucidate the mechanism of the action of cocaine on MRF neurons.

How the neurophysiological effect of cocaine on MRF neurons is related to behavioral antinociception is unclear. Because bulbospinal neurons can inhibit nociceptive inputs ${ }^{2,9,10}$, cocaine-induced increases in spontaneous activity would be expected to produce inhibition of

\section{REFERENCES}

1 Azzaro, A.J., Ziance, R.T. and Rutledge, C.O., The importance of neuronal uptake of amines for amphetamine-induced release of ${ }^{3} \mathrm{H}$-norepinephrine from isolated brain tissue, $J$. Pharmacol. Exp. Ther., 189 (1974) 110-118.

2 Basbaum, A.I. and Fields, H.L., Endogenous pain control mechanisms: review and hypothesis, Ann. Neurol., 4 (1978) 451-462.

3 Belczynski, C.R., Pertovaara, A., Morrow, T.J. and Casey, K.L. Cocaine suppression of medial thalamic nociceptive responses in the rat, Soc. Neurosci. Abstr., 14 (1988) 853.

4 Bowsher, D., Mallart, A., Petit, D. and Albe-fessard, D., A bulbar relay to the center median, J. Neurophysiol., 31 (1968) 288-300.

5 Brodal, A., The Reticular Formation of the Brainstem: Anatomical Aspects and Functional Correlations, Charles C. Thomas, 1957, 44 pp.

6 Burton, H., Somatic properties of caudal bulbar reticular neurons in the cat (Felis domestica), Brain Research, 11 (1968) 357-372.

7 Casey, K.L., Somatic stimuli, spinal pathways, and size of cutaneous fibers influencing unit activity in the medial medullary reticular formation, Exp. Neurol., 25 (1969) 35-56.

8 Eccles, J.C., Nicoll, R.A., Taborikova, H. and Willey, T.J., Medial reticular neurons projecting rostrally, J. Neurophysiol., 38 (1975) 531-538.

9 Fields, H.L., Basbaum, A.I., Clanton, C.H. and Anderson, S.D., Nucleus raphe magnus inhibition of spinal cord dorsal horn neurons, Brain Research, 126 (1977) 441-453. nociceptive transmission through the spinal dorsal horn. The persistence of inhibitory, noxious-evoked responses in MRF neurons after cocaine precludes the possibility of a generalized spinal inhibition. A descending bulbospinal inhibitory effect of cocaine must be selective for excitatory transmission. Pertovaara (personal communication) has shown that cocaine suppresses the nociceptively evoked activity without altering spontaneous activity of both nociceptive-exclusive and wide-dynamic-range spinal dorsal horn neurons that project to the reticular formation. This neuronal response suppression may contribute to the reductions in nociceptive responsiveness observed in the MRF. Alternatively, the behaviorally relevant action of cocaine may be due to its effect on rostrally projecting MRF neurons. Indeed, we currently have evidence for cocaine-induced suppression of medial thalamic nociceptive responses ${ }^{3}$.

The evidence thus far supports a supraspinal, dopaminergic mechanism for cocaine-induced antinociception that may involve known bulbospinal inhibitory systems. Cocaine promises to be a useful tool for probing the neurophysiological mechanisms underlying pain and analgesia. The non-opiate nature of its antinociceptive action may help us to test and expand existing theories regarding pain perception and processing.

Acknowledgements. This work was supported by a grant from the Veterans Administration and a Bristol Myers Award, C.R.B. by an NSF Predoctoral Fellowship and A.P. by the Fogarty Foundation.

10 Fields, H.L. and Basbaum, A.I., Endogenous pain control mechanisms. In P.D. Wall and R. Melzack, (Eds.), Textbook of Pain, Churchill Livingstone, Edinburgh, 1984, pp. 142-152.

11 Fuller, J.H. and Schlag, J.D., Determination of antidromic excitation by the collision test: problems of interpretation, Brain Research, 112 (1976) 283-298.

12 Fuxe, K.B., Hamberger, B. and Malmfors, T., The effect of drugs on accumulation of monoamines in tubero-infundibular dopamine neurons, Eur. J. Pharmacol., 1 (1967) 334-341.

13 Hadfield, M.G., Mott, D.E.W. and Ismay, J.A., Cocaine: effect of the in vivo administration on synaptosomal uptake of norepinephrine, Biochem. Pharmacol., 29 (1980) 1861-1863.

14 Harris, J.E. and Baldessarini, Uptake of $3 \mathrm{H}$-catecholamines by homogenates of rat corpus striatum and cerebral cortex: effects of amphetamine analogues, Neuropharmacology, 12 (1973) 669-679.

15 Harrison, P.W., The intravenous use of cocaine. Report of a case, Boston Med. Surg. J., 164 (1911) 151.

16 Ito, M., Udo, M. and Mano, N., Long inhibitory and excitatory pathways converging onto cat reticular and Dieters' neurons and their relevence to reticulofugal axons, $J$. Neurophysiol., 33 (1970) 210-226.

17 Kast, L. and Meltzer, S.J., On the sensibility of abdominal organs and the influence of injections of cocaine upon it, Med. Rec., 70 (1906) 1017-1019.

18 Koe, B.K., Molecular geometry of inhibitors of the uptake of catecholamines and serotonin in synaptosomal preparations of the rat brain, J. Pharmacol. Exp. Ther., 199 (1976) 649-661.

19 LeBars, D., Dickenson, A.H. and Besson, J.-M., Diffuse noxious inhibitory controls (DNIC). I. Effects on dorsal horn 
convergent neurons in the rat, Pain, 6 (1979) 283-304.

20 Lin, Y., Morrow, T.J., Kiritsy-Roy, J.A., Terry, L.C. and Casey, K.L., Cocaine: evidence for supraspinal, dopaminemediated, non-opiate analgesia, Brain Research, 479 (1989) 306-312.

21 Magni, F. and Willis, W.D., Identification of reticular formation neurons by intracellular recording, Arch. Ital. Biol., 99 (1963) 33-67.

22 Misra, A.L., Pontani, R.B. and Vadlamani, N.L., Stereospecific potentiation of opiate analgesia by cocaine: predominant role of noradrenaline, Pain, 28 (1987) 129-138.

23 Morrow, T.J., Improved technique for recording single unit activity in awake animals, Brain Res. Bull., 5 (1980) 91-93.

24 Morrow, T.J. and Casey, K.L., Suppression of bulboreticular unit responses to noxious stimuli by analgesic mesencephalic stimulation, Somatosens. Res., 1 (1983) 151-168.

25 Paxinos, G. and Watson, C., The Rat Brain in Stereotaxic Coordinates, Academic Press, New York, 1986.

26 Pertovaara, A., Belczynski Jr., C.R., Morrow, T.J. and Casey, K.L., The effect of cocaine on spinal nociceptive reflex activity in the rat, Brain Research, 43 (1988) 286-290.

27 Pitts, D.K., Udom, C.E. and Marwah, J., Cardiovascular effects of cocaine in anesthetized and concious rats, Life Sci., 40 (1987) $1099-1111$

28 Pitts, D.K. and Marwah, J., Cocaine modulation of central monoaminergic transmission, Pharmacol. Biochem. Behav., 26 (1987) 453-461.

29 Ritter, C., Total Anasthesie durch Injektion von Cocain in die Vene, Berliner Klin. Wochenschr., 46 (1909) 1701-1702.

30 Ross, S.B. and Renyi, A.L., Inhibition of the uptake of tritiated 5-hydroxytryptamine in brain tissue, Eur. J. Pharmacol., 7 (1969) 270-277.

31 Swanson, L.W., The locus coeruleus: a cytoarchitectonic, Gotgi and immunohistochemical study in the albino rat, Brain Research, 110 (1976) 39-56.

32 Wolstencroft, J.H., Reticulospinal neurones, J. Physiol. (Lond.), 174 (1964) 91-108.

33 Yang, J.C., Clark, W.C., Dooley, J.C. and Mignogna, F.V., Effect of intranasal cocaine on experimental pain in man, Anesth. Analg., 61 (1982) 358-361. 